\title{
Complications of pregnancy: premature rupture of membranes,chorioamnionitis, amnioinfusion, prenatal administration of surfactanct
}

\begin{abstract}
It is defined as premature rupture of membranes (RPM) to the leak out of amniotic fluid by genitalia before the onset of labour. ${ }^{1}$ It's one of the most frequent in obstetric practice diagnoses and their implications are quite different as this fact occurrence of term or before it. It presents a serious problem because of their difficult prediction and the mechanisms involved are still unknown. We analyzed in this chapter the physiopathology, associated complications and different approaches to treat the picture.
\end{abstract}

Keywords: premature rupture of membranes, pregnancy, oligoamnios, prenatal surfactanct
Volume 4 Issue I - 2018

\author{
Ricardo Illia,' Claudio Solana, ${ }^{2}$ Lobenstein \\ $\mathrm{G},{ }^{3}$ Uranga Imaz M, ${ }^{3}$ Manrique G, ${ }^{3}$ Fiameni \\ $\mathrm{F}^{3}$ Guallan $\mathrm{F}^{4}$ \\ 'Chief of Obstetric Service at Hospital Alemán, Buenos Aires, \\ Argentina \\ ${ }^{2}$ Chief of Intensive Neonatal Care Unit, Hospital Alemán, \\ Argentina \\ ${ }^{3}$ Staff of Obstetricians, Obstetrics Service Hospital Alemán, \\ Argentina \\ ${ }^{4}$ Chief of Residents, Obstetrics \& Gynecology Service, Hospital \\ Alemán, Argentina
}

\begin{abstract}
Correspondence: Ricardo Illia, MD, FACOG, Buenos Aires University, Chief of Obstetric Service at Hospital Alemán, Buenos Aires,Argentina, Email rhillia@gmail.com
\end{abstract}

Received: January 14, 2018 | Published: January 29, 2018

\section{Introduction}

It is defined as premature rupture of membranes (RPM) to the leak out of amniotic fluid by genitalia before the onset of labour. ${ }^{1}$ It's one of the most frequent in obstetric practice diagnoses and their implications are quite different as this fact occurrence of term or before it.

In the first case, occur in RPM approximately $10 \%$ of patients. While there is risk of infection to the mother and the fetus, this risk is not as important as when the RPM takes place before the term. Usually, between $70 \%$ and $80 \%$ of the patients at term with RPM spontaneously initiate labour within the subsequent 24 hours and those that did not begin spontaneously,the induction of labor is indicated within 12 hours.

Some authors prefer at term to shorten this period of waiting (latent period) starting the induction of labor between 12 and 24hours after produced the RPM. ${ }^{2}$ Before the term of pregnancy, RPM occur with a variable frequency. According to the authors this frequency varies between 5 and 30 percent of preterm births and is a significant contributor to perinatal mortality. ${ }^{3-6}$

It presents a serious problem because of their difficult prediction and the mechanisms involved are still unknown. RPM before the term is associated with approximately $50 \%$ of histological chorioamnionitis (5-6). Romero et al. ${ }^{7}$ have found a frequency of chorioamnionitis associated with RPM between 15 and $100 \%$ of the cases studied ${ }^{7-8}$ as well as these patients had a higher incidence of postpartum and postoperative infection..$^{9-10}$ At the place of rupture, it can be observed a marked inflammatory reaction and the fact to recover bacteria from amniotic fluid in up to $80 \%$ of the cases, suggest a strong association between RPM before the term, preterm birth and infection. However not all cases of RPM before the term are of infectious origin, and may be involved other pathophysiological ${ }^{11}$ processes. These processes include maternal or fetal vascular injury (up to $20 \%$ of cases of RPM) and in $13 \%$ of the population studied by Arias et al. they did not found any type of placental or ovular injury associated with RPM.

Complications associated with RPM before the term, as well as fetus-maternal-neonatal infection, include prematurity generally and in particular perinatal asphyxia, respiratory distress of variable intensity, hypoplasia and pulmonary dysplasia, ventricular hemorrhage, necrotizing enterocolitis, etc, all entities that are common in the preterm new born. ${ }^{12}$

\section{Etiology}

Apparently, the membranes have some elastic properties. Depending on the content of elastin, membranes have the possibility of adapting to a deformation with recovery of its original state. ${ }^{13}$ Depending on demand of these properties, or endogenous pressure by contractions or by exogenous pressure by trauma or infection, membranes are weakened by increasing the chance of rupture. ${ }^{14}$ It has been reported that the content of collagen is lower in patients with RPM that in those without RPM, so that a decrease in the content of collagen in membranes could predispose the RPM. ${ }^{15}$ Other authors suggest that there would be no difference in the content of collagen in the membranes of patients with and without RPM. They propose that in the case of RPM, there would be an increase in collagenolytic activity at membranes. ${ }^{14}$ Fortunato et al. ${ }^{5}$ proposed that collagenolytic enzymes may play a role in infection-associated RPM and preterm birth. It seems to exist a mechanism of cellular remodeling in membranes products of conception through the activity 
of matrix metalloproteinases during pregnancy to resist the increase in intrauterine pressure and volume.

A significant decrease in the content of collagen towards the end of gestation would allow the possibility of colagenolysis membrane products of conception. This is evidence that the rupture of the membranes is not always associated to changes in uterine contractility. It has been proposed that the surfactant present in the amniotic cavity would provide a lubricating effect. At early gestational ages, the absence of surfactant in the amniotic fluid could facilitate the RPM. ${ }^{16}$ The infection as an important etiological factor in RPM has recently acquired importance before the term. ${ }^{17-18}$ By cultured from amniotic fluid have been shown germs in a significant number of patients with RPM, including Neisseria gonorrhea, Group B streptococcus, Bacteroides, Trichomonas, Chlamydia, Mycoplasma, Ureaplasma, Gardnerella and Candida Albicans among others. ${ }^{19-20}$

Several studies have examined the role of membranes infection by Chlamydia and its association with RPM. It has been attributed to Chlamydia trachomatis an etiologic role regarding preterm birth and RPM. After infection, could increase the amount of leukocytes with subsequent stimulation of phospholipase A2 by lysing macrophages, finally generating the activation of the mechanism of cyclooxygenase, prostaglandins increase. This event is related to the increase in uterine activity promoting RPM and preterm birth. ${ }^{21}$ In addition to the role assigned to Chlamydia with RPM, it is estimated that 25 to $50 \%$ of infants born vaginally of colonized mothers suffer conjunctivitis and $10-20 \%$ will suffer pneumonia by Chlamydia. ${ }^{22}$

In relation to these infections it has been described the production and release of infected cells by interleukins and tumor necrosis factor The discovery of these elements in the amniotic fluid lend support to an inflammatory process in evolution. In fact, these substances are taken into account by some authors as elements of early diagnosis of the ovular infection. ${ }^{23}$

The Interleukin 6, is an inflammatory cytokine produced by several types of cells including monocytes, macrophages, amnion, chorion, and decidual cells. It integrates a group of inflammatory mediators of tumor necrosis factor and Interleukin 1 and they are produced by macrophages and decidual cells in response to a wide variety of bacteria and bacterial products. They are found in high concentrations in amniotic fluid in the presence of infection and is thought to have an important role in the mechanisms the preterm delivery associated pathophysiologically with RPM. ${ }^{24}$ It is thought that this cytokine (IL-6) plays an important role in the intrauterine infection response and reported as high in the amniotic fluid of patients with RPM and microbial invasion of the amniotic cavity. ${ }^{12}$ Recently it has been reported that the interleukin 8 was detected in the urine of 91 of 104 patients with RPM, with high values in cases of severe intra-amniotic infection. ${ }^{25}$

Early termination of pregnancy, inadequate fetal development, bleeding, the threat of preterm delivery and RPM, could result from the colonization of upper genital tract by chlamydia. The released cytokines may generate inflammatory alterations in membranes predisposing to the RPM. It has also shown a relationship between infection by Neisseria gonorrhea and preterm birth associated with RPM. ${ }^{26}$ Several authors have documented the association between infection and preterm birth-RPM mediated by Bacteroides ${ }^{27}$ and Gardnerella Vaginalis. ${ }^{28}$ Recent studies have reported an association between bacterial vaginosis and adverse outcomes of pregnancy as preterm birth, RPM and peripartum infections including intraamniotic infections. Bacterial vaginosis is associated with a characteristic pattern of organisms in the vagina as high concentrations of Gardnerella Vaginalis, Mycoplasma species and anaerobic bacteria that are associated with a decrease in the concentration of Lactobacilli. The germs found in the amniotic fluid of patients with ovular infection pattern is similar to the associated with presence of bacterial vaginosis. ${ }^{29}$

It is described clearly the association between Streptococcus Group B (EB) and preterm birth-RPM. The EB is the most common cause of neonatal sepsis in the USA. ${ }^{30}$ It occurs in 2 to 4 per 1,000 live newborns $\mathrm{s}^{31}$ and risk factors are a significant genital colonization of the mother, preterm delivery, RPM and intrapartum maternal fever.

In the study by Romero et al. ${ }^{20}$ the most frequently associated germen with infection of membranes was Mycoplasma. RPM patients had a higher frequency of infection by Mycoplasma than patients with preterm labor but with intact membranes. Indirectly the vaginal $\mathrm{PH}$ has been linked with RPM in those patients with not adequately acidic vagina. This predisposes to vaginal infections by the above mentioned germs with the consequent possible rise of the same to the ovular cavity.

Ernest et al. ${ }^{32}$ measured the vaginal PH of 115 patients with risk of preterm delivery. They found an increase of 3times probability of RPM between patients with $\mathrm{PH}>4.5$ that in which $\mathrm{PH}$ was lower. Riedewald et al. ${ }^{33}$ also observed a high correlation between increased vaginal $\mathrm{PH}$ and preterm delivery.

Some authors stated about a relationship between higher incidence of prematurity-RPM in patients with nutritional deficiencies. Perhaps the most important element is Zinc, which has been attributed antibacterial and anti-viral properties in the amniotic fluid. ${ }^{34}$ It seems that zinc combined with other elements is one of the main antibacterial mechanisms of amniotic fluid. Smoking is also associated with increased frequency of RPM. Relationship between higher probability of RPM and smoking more than 10 cigarettes a day ${ }^{35}$ has been found.

Harger et al. $^{36}$ analyzed the relationship between RPM and smoking, and vaginal bleeding. Previous prematurity, smoking, and vaginal bleeding were significantly associated with RPM. They proposes a chronic evolution of placental abruption, as a cause of the bleeding so that blood would produce uterine irritability with consequent increase of the intra-amniotic pressure, contractions and placental separation.

\section{Istmico-cervical incompetence also has a role in birth preterm-rpm}

The approach would be that the progressive cervical dilatation contacted membranes with vaginal flora. So, there would be a rise of germs causing infection of membranes and the final expulsion of the uterine contents. ${ }^{37}$

It should be noticed the similarity between the obstetric histories of perinatal losses to repeat at early gestational ages between upper genital tracts colonized patients and patients with genuine istmicocervical incompetence. This similarity suggests that it has been offered as a therapeutic alternative the cervical cerclage to patients actually presenting repeated ovular infection. And this assumption is based on that there have been cases of patients who after a pregnancy to term with a cerclage, have come to end in the next pregnancy without banding. 
Anyway, it is interesting to note that cervical cerclage is a valid alternative as adjuvant in the handling of these situations since the cervical closure, keeps the mucous plug in place insulating the ovular cavity of the vaginal flora. Regarding the ascending infection, it is interesting to remember that many years ago, Naye in Africa, intended to empirically that people predisposed to ovular infection to by background, maintained sex during pregnancy with condom to avoid ascending infection.

In our environment, our group has researched to much this subject in patients with perinatal losses repeated by amnionitis at gestational ages usually less than 30 weeks. We have designed a protocol that includes the prophylactic cerclage around 14weeks to avoid infection upward and simultaneously the administration of azithromycin until 34 weeks to avoid potentially colonized tubas descending infection. This Protocol is associated with more than $90 \%$ of success in the reduction of the prematurity of infectious cause mainly in patients with perinatal loss by ovular infection before 30 weeks. ${ }^{38}$

\section{Pathophysiology}

The germs could access the amniotic cavity using one of the following mechanisms:

a. ascending from vagina and cervix.

b. Hematogenous dissemination through the placenta.

c. retrograde spread from the peritoneal cavity through the fallopian tubes.

d. accidental introduction through invasive procedures such as amniocentesis, cordocentesis, aspiration of villus sampling.

There is evidence that the most frequent way is the ascendant. This evidence is based on:

i. The histological chorioamnionitis is more common and severe in the site of rupture of membranes than in other locations.

ii. The bacteria identified in case of congenital infection are similar to those found in the genital tract.

iii. In twin pregnancy, the histological chorioamnionitis is more common in the first fetal sac and its existence only in the second sac has not been demonstrated.

Romero has proposed four stages that will lead to intrauterine infection. ${ }^{39}$

The first stage consists of an excessive growth of facultative germs or the presence of pathological germs in the vagina or the cervix. Bacterial vaginosis can be one of the manifestations of stage 1 .

Once the germs gain access to the uterine cavity and are located in the decidua, we are in stage 2. A deciduitis appears and then the chorioamnionitis.

Infection could invade fetal vessels (coriovasculitis) or enter the amniotic cavity (amnionitis) determining stage 3. Once in the amniotic cavity, the germs can access the fetus (stage 4). The aspiration of amniotic fluid infected by the fetus can lead to congenital pneumonia and eventually spread causing bacteremia and sepsis.

The mechanisms responsible for the RPM can be associated to ascending infection. Located in the coriodecidual union, infection can lead to RPM and can also result in a stage 3.
The germs that were more commonly found in the study of Romero in patients with intact membranes and preterm labour were Ureaplasma urealitycum, Fusobacterium and Mycoplasma hominis. The difficulties in defining the role of Chlamydia trachomatis in the etiology of microbial invasion and intrauterine infection may be due to problems in isolating the microorganism with traditional media. Also, patients with positive amniotic fluid culture in general had no clinical evidence of infection, but developed a higher incidence of chorioamnionitis, and were refractory to tocolysis and presented RPM more frequently than patients with negative culture.

Recent data show that patients with RPM and severely reduced amniotic fluid have higher incidence of intraamniotic infection. There is evidence that treatment of asymptomatic patients with positive cervical cultures for Chlamydia, reduces the risk of presenting RPM. ${ }^{38,40,41}$ The RPM mechanism involves the induction of an acute inflammatory reaction and the subsequent initiation of release of prostaglandins and cytokinas waterfall.

In addition, this body acts as an intracellular parasite and could lead to destruction of amniocites. ${ }^{22}$ In this study of Ville et al. ${ }^{22}$ Chlamydia was identified in $7.4 \%$ of pregnant women with RPM and in all cases there were concomitant infection of the lower genital tract with the same germ, suggesting an ascending via related with the acquisition of infection. The finding that intra-amniotic infection occurred only in 6 of 20 patients with cervical infection, suggests that as with other pathogens, lower genital tract colonization is not enough itself to condition intrauterine infection. The cervical mucus and amniotic fluid have antibacterial properties, and studies in vitro have shown that amniotic fluid may inhibit Chlamydia cellular inclusions in cell cultures of McCoy.

The biosynthesis of prostaglandins may be stimulated by bacterial factors or the host in response to the presence of germs. The most widely accepted explanation for the onset of labour in the presence of infection is bacterial products to stimulate the synthesis of prostaglandins. Several researchers have shown that bacterial products are a source of phospholipase A2 and C that would stimulate the production of prostaglandins. ${ }^{40}$

Romero, proposes a model in which the initiation of labor in the presence of infection is controlled by the host. In the case of maternal infections such as systemic pyelonephritis or localized deciduitis, infections can trigger the delivery via the monocytemacrophage system. According to this scheme, the preterm delivery can be considered an event that occurs when the uterine or maternal environment is hostile to fetal well-being.

The cytokines can participate in the RPM. The pathophysiology of the RPM could be similar to the preterm delivery. The bacterial infection can induce the release of proteases from macrophages and other cells, which degrade the membranes and cause breakage. The reason why some intrauterine infections end in preterm birth and others in RPM must still be clarified. Rosemary considers these facts as different expressions of the same problem: activation of macrophages in the host system. The infection is important, but not the only link of this chain of events.

From the histological point of view, we can find different types of placental injury associated with RPM:11

A. Inflammatory acute injury characterized by the presence of infiltration of polymorphonuclear leukocytes of varied length and 
severity, with or without necrosis, fetal membranes and subchorionic layer, with or without compromise of the umbilical cord.

B. Native vascular lesions in the absence of inflammatory changes: placenta hypoplastic, multi-infarct, intervellous thrombosis, thrombosis or narrowing of spiral arterioles of placenta and abruptio placentae confirmed by identification of clots in the maternal surface.

C. Vascular lesions fetal (fetal thrombotic vasculopathy) characterized by thrombosis of fetal vessels resulting in areas of avascular villi with fibrosis of the stroma.

D. Mixed injuries, placentas exhibiting acute inflammatory lesions and vascular lesions.

E. Normal placentas at macro and microscopy.

In this study of Arias et al. ${ }^{11}$ the most numerous group presented acute inflammatory lesions (43.4\%). In the majority of these patients (64.7\%) RPM arose before 30weeks, perinatal mortality amounted to $18.6 \%$ and the incidence of positive cultures was $28.4 \%$. The majority of these patients had a period of latency greater than 48 hours.

Maternal or fetal vascular lesions were observed at $20.4 \%$. In the majority of patients who presented mixed injuries and acute inflammation, RPM took place at early gestational ages. This study suggests that all patients with RPM are not equal and that they can be classified and separated into different histological groups with distinct clinical features and prognostic differences.

\section{Clinical picture}

When the RPM is clearly evident, the patient clearly refers leakage of liquid from their genitals and usually it could be proven placing a speculum and seen amniotic fluid in vaginal cul-de-sac. It is very important for the physician not to leave doubts about the diagnosis of RPM since its presence or absence changes dramatically the therapeutic proposal and potential complications for mother and fetus. In the case of observed maternal fever $\left(38^{\circ} \mathrm{C}\right.$ or more) and two of the following signs: uterine irritability, fetal tachycardia (160 beats per minute or more), maternal tachycardia (100 beats per minute or more), purulent vaginal discharge, white blood cell count equal to or greater than 12,500 , more than $85 \%$ of neutrophilia, the diagnosis of chorioamnionitis ${ }^{11}$ is imposed.

However, none of these signs is totally categorical (with the exception of the presence of pus into vagina coming from the uterine cervix). From the practical point of view, we must suspect an ovular infection with or without RPM in the presence of uterine contractility that does not respond to tocolysis in the usual terms. This is the sign of greater sensitivity and as already mentioned, the probability of ovular infection is greater in the case of RPM (or without RPM) in which there is a severe oligohydramnios detected by ultrasound. In cases in which the picture is doubtful, the clear reference by the patient's fluid loss by genitalia associated with decrease of the amount of amniotic fluid ultrasound observation must be taken into account as suggestive of RPM.

\section{Diagnostics}

We will list the elements that orient themselves or claim the diagnosis by way of summary:
I. A clear observacion of amniotic fluid in vaginal cul-de-sac.

II. If is not clear that the observed material is amniotic fluid, take a sample to see the crystallization in fern leaves.

III. If observed material contains blood, washing the vagina with dextrose $5 \%$ (sodium chloride also crystallizes) and then try the test a sample for crystallization.

IV. Use of nitrazina test to verify the change of coloration to get in contact with the vaginal content (if there is amniotic fluid in vagina, its $\mathrm{PH}$ is less acid producing change in staining).

V. Evaluate the volume of amniotic fluid by ultrasound. Its decline associated with the methods described in C and D, strongly suggest the diagnosis.

The confirmation of the points A and B, confirm the diagnosis.

\section{Differential diagnostics}

There are few situations that can confuse the diagnosis, but there are. The patient can refer she feels the elimination of fluid from vagina. The vaginal examination does not show the presence of amniotic fluid in vagina and crystallization is negative. If in addition the volume of amniotic fluid by ultrasound has not altered, most likely is that it is vaginal discharge which is appropriate to evaluate or treat, but is not an RPM.

In the second instance, sometimes by some movement or fetal rearrangement, the maternal bladder is compressed and if it is full of urine, may have a partial emptying referred by the patient as a sudden elimination of liquid by genitals. The cessation of all loss once surpassed the episode induce to think in favour of probable loss of urine with no amniotic fluid. When the RPM has occurred, the patient continues losing fluid without interruption at any possition.

\section{Studies to diagnose ovular infection}

At least one-third of the patients presenting RPM before the term have microbial invasion of the ovular cavity. An early diagnosis and of certainty of ovular infection is important given the complications that these situation could produce in the preterm fetus or newborn, related or not with the amnionitis.

In the study by Romero et al..$^{20}$ they found that the amniocentesisbirth interval was lower in patients with positive amniotic fluid culture. The rate of neonatal complications was higher in patients with ovular infection. These data represent a strong argument about the importance of ovular infection as risk factor of adverse perinatal outcome.

In this study the most frequently isolated germ was Mycolpasma Hominis, made more common in patients with threat of preterm delivery with or without RPM. An interesting observation in this study was the importance of the amount of germs inoculated as a determinant of changes in glucose contents in amniotic fluid, count of white blood cells and concentration of IL-6. The results of the study show that IL-6 concentrations were better predictors of the outcome of the cultivation of amniotic fluid, the duration of the interval admission-delivery and morbidity and mortality perinatal that Gram stain, count of white blood cells in amniotic fluid and also in amniotic fluid glucose concentrations.

A microbial invasion of recent data as that which probably occurs 
in the case of RPM may be sufficient to produce a positive culture but not sufficient stimulus to produce a complete response of the host. On the other hand, the ovular infection with intact membranes has a longer period of latency, allowing a complete immune and inflammatory response. Gauthier et al. ${ }^{19}$ the diagnostic value of these measurements also were studied. Their results show that leukocyte esterase activity and amniotic fluid glucose concentration were significantly more sensitive than the Gram stain to detect positive cultures of amniotic fluid.

But, none of these tests had power enough to predict the results of the culture, so the authors advised not to base clinical decisions only on the basis of these results. Murtha et al. ${ }^{12}$ also studied levels of interleukins finding that Interleukin-6 concentrations were elevated in patients with RPM and clinical or histological chorioamnionitis.

Recently Hsu et al. ${ }^{42}$ assessed metabolites of nitric oxide and Guanosine monophosphate as indicators of ovular infection. The nitric oxide (no) is a free radical unstable easily oxidizable to nitrates and nitrites in few seconds. It can exert cytotoxic and cytostatic effects in the white cells with inflammatory, antibacterial, and antiviral actions. Stiemer et al. ${ }^{43}$ analyzed the possibility of dose interleukin 8 in maternal urine. The IL- 8 was detected in the urine of 91 of 104 patients with RPM, with values exceeding $1,000 \mathrm{ng} / \mathrm{L}$ in patients with severe intra-amniotic infection. According to the authors the routine measurement of IL-8 in urine provides a simple, low-risk method for the detection of intraamniotic infection.

Unfortunately these tests are not available to the physician in our country with a few exceptions. But should also be taken into account that none has enough sensitivity as for their ability by itself to predict ovular infection, so for our medicine, these studies are of doubtful utility and would increase our costs too. To our flow chart of action before cases with RPM, the refractoriness to tocolysis continues to be the most important indicator of probable ovular infection.

However, in 1986 Vintzileos et al ${ }^{44}$ reported that daily biophysical profile was better than amniocentesis to predict ovular infection in patients with RPM. They found in the study that around $80 \%$ of the cases associated with infection presented a non reactive antepartum fetal monitoring, while cultures were positive in $60 \%$ of the sample. The controversy arises when the RPM has no clear clinical signs of ovular infection. In these cases, some researchers have proposed the use of amniocentesis to obtain a sample of amniotic fluid. The problems associated with this approach are the impossibility of obtaining liquid in more than half of these patients, the invasiveness of the procedure with its inherent risks and the lack of a strong correlation between the presence of bacteria in the sample and the presence of fetalneonatal sepsis. The presence of bacteria in amniotic fluid has been correlated well with the development of amnionitis and postpartum endometritis, but not fetal-neonatal sepsis. This is not surprising given that the presence of bacteria in amniotic fluid reflect what happens in the intrauterine environment but not the fetal condition.

In previous studies, the same Vintzileos ${ }^{45,46}$ stated that the biophysical profile was an early predictor of infection in patients with RPM. The best predictor was the full profile, where the first manifestations of fetal compromise were not reactivity followed by loss of fetal breathing movements. According to Vintzileos studies, the effectiveness of the biophysical profile in predicting complications associated with ovular infection is superior to the amniocentesis. Also Vintzileos has studied the value of the analysis of the amniotic fluid volume in perinatal outcomes of patients with RPM. Cases of severe oligohydramnios, defined as the presence of lakes of less than $1 \mathrm{~cm}$ of vertical length, associated with an increased frequency of variable decelerations, low Apgar Score at the minute and fifth of life, perinatal death and ovular infection.

The rules of follow-up for patients with RPM in the Hospital Alemán of Buenos Aires, contain guidelines based on the antepartum fetal monitoring, fetal breathing movements count, and the evolution of the volume of amniotic fluid. Both the reactivity absence as the non-presence of fetal breathing movements, would induce us to suspect the presence of ovular infection. Obviously we do not propose defining conduct to a non-reactive monitoring and less at this gestational age, but in the case of non reactivity and the finding of the presence of respiratory movements is a reassurance of the fetal status. In terms of the assessment of the volume of amniotic fluid, we are working on the possible role of amnioinfusion during pregnancy and before delivery, as an alternative of value to improve fetal habitat conditions, decrease the frequency of fetal deformation and basically to try to prevent the fetal pulmonary hypoplasia. We have performed certain procedures at the Hospital and also in the private population of patients with perinatal promising results that will be reported to the scientific societies as soon as we can.

\section{Chorioamnionitis}

The acute chorioamnionitis is a little frequent but a serious picture, occurring in around 0.5 to $2 \%$ of pregnancies. ${ }^{47}$ Although there is general agreement that the treatment consists of antibiotics and uterine evacuation, there is no general agreement as to the time of both treatments. Sperling et al. ${ }^{48}$ reported significantly higher incidence of neonatal sepsis in newborns whose mothers were treated with antibiotics after the clamping of the umbilical cord that patients who received antibiotics before clamping. Gilstrap et al. ${ }^{47}$ found that administration of antibiotics to the mother during labour may result in a decrease in the frequency of neonatal sepsis.

Ovular infection can occur through intact membranes with Ureaplasma urealyticum and Mycoplasma hominis, ${ }^{49}$ resulting in a silent, chronic infection, characterized by an intense inflammatory response. There may also be the infection in case of RPM. With regard to the cause of perinatal death, morphological causes in autopsies of almost two-thirds of fetuses have not been documented. In the study of Quinn, ${ }^{49}$ amnionitis was observed in $57.6 \%$ of 33 perinatal deaths and $70.8 \%$ of positive placentas for chorioamnionitis were observed in cases without a cause of fetal death versus $22.2 \%$ of associated deaths with other concomitant causes.

We may find ourselves with cases in which there is cervix of purulent fluid output (take material for cultivation) where the diagnosis is absolutely clear and the most critical issue is the way of birth, but the majority of the cases are those with prolonged rupture of membranes, indistinct signs of laboratory, with repeated episodes of paraenteral tocolysis, which as has been expressed the main sign suggesting the presence of an ovular infection is refractoriness to the tocolysis.

In front of a clear diagnosis or strong suspicion of chorioamnionitis, behavior is uterine evacuation. We should privilege be vaginally way since surgery will get in touch with the peritoneal cavity maternal intrauterine germs and is prudent before performing a caesarean section, put in balance the risks for both the fetus and mother assessing 
gestational age, estimated weight, parity and maternal age, survival potential at that gestational age in the center that will take place the procedure and possibilities of referral to a Center with greater resourses to the assistance of babies of very low weight.

Our point of view is to maintain the antibiotic that the patient was receiving for its condition of RPM and discuss any possible changes with neonatologists and if possible with infectiologists. The position of the obstetrician perinatologist should be careful and while the debate focused on the chances of survival for the fetus, not to forget the situation of the mother who will not benefit from empirical or aggressive behaviors. All interventional behavior must be properly grounded and if there are possibilities and time (not common in these situations) submit conduct debate on a Bioethic Committee.

An intervention of the most importance is the administration of corticosteroids to accelerate fetal lung maturation. From our point of view, we do not believe that before an infection already installed the maternal condition impair by administering a dose of steroids and it is proven that after four hours of applied, steroids begin to have effect on lung maturation. ${ }^{50}$ It is an extremely critical situation, so, any action which could potentially generate benefit current or subsequent to neonatal survival we believe that it is justified.

Produced the birth, if the route was vaginal, and we have identified germ; it is advisable to keep the antibiotic scheme, which will be subject to modification according to the evolution of the patient or the result of crops. If the via is in abdominal, is convenient to protect the para colonic places with compresses to try to prevent the spread of the uterine contents and make a good uterine closure, and consider as appropriate the placement of drainage type rubber tube in cul-de-sac of Douglas.

The fact of having led to the opening of the visceral peritoneum, has put the patient at risk of a peritonitis. For that reason the drains are important and the antibiotic scheme should be changed to double or triple always including one who is probably effective for the amnionitis germs action (Mycoplasma, ureaplasma, chlamydia).

\section{Administration of corticosteroids}

In 1994 the consensus achieved by the National Institutes of Health in USA informed recommendations on the use of corticoids antenatal to induce fetal lung maturation. Conclusion of this consensus, communicates that although it seems to have a discrete increase in maternal and neonatal infection rates in cases where administered corticosteroids, this risk is minimal compared with the benefits obtained with antenatal administration..$^{50}$

However, the Committee on Obstetric Practice for the American College of Obstetricians and Gynecologists respected all the recommendations emanating from the Consensus with the exception of those concerning the use of corticosteroids in the case of RPM. ${ }^{51}$ The manifest Committee needed further studies with respect to the use of corticoids antenatal patients with RPM.

The consensus has been achieved on the data provided on the retrospective analysis of four large databases and subsequently published by Wright et al. ${ }^{52}$ which show that in pregnancies with RPM before term, the antenatal corticosteroid use reduced the likelihood of neonatal death around 50\%(OR 0.43, CI 0.37-0.50). Similar findings were obtained Gunkel \& cols. ${ }^{53}$

The meta analysis by Crowley ${ }^{50}$ and considered for Consensus, indicated that the use of antenatal corticoids was associated with a reduction of more than $50 \%$ of (OR 0.44$)$ respiratory distress syndrome. Also in this review, no association was observed between use of corticosteroids and an increase in perinatal infection.

A retrospective analysis of the National Institute of Child Health (USA) on a population of newborns of very low birth weight (1500g or less) showed that antenatal exposure to corticosteroids was associated with a lower frequency of intraventricular hemorrhage (OR $0.53, \mathrm{CI}$ 0.44-0.64) and there was no increase in the incidence of neonatal sepsis (OR 0.58, CI 0.34-0.98) ${ }^{54}$ After reviewing the data, the panel concluded that the potential benefits in reducing the frequency and severity of intraventricular hemorrhage associated with the use of antenatal corticoids were superior to the potential risk of increasing the rate of neonatal sepsis in pregnancy preterm labor with RPM. The effectiveness of antenatal steroids in reducing the frequency of intraventricular hemorrhage in newborns of very low birth weight with RPM had been demonstrated previously by Morales et al. ${ }^{55}$

Recently, Chapman et al. ${ }^{56}$ relativized the benefits of the administration of antenatal corticoids in case of extreme prematurity (birthweight of $1,000 \mathrm{~g}$ or less) not observing a decrease in the frequency of intraventricular hemorrhage.

Assuming a reduction in the frequency of severe intraventricular hemorrhage of $13 \%$ to $8 \%$ as seen in data from the Consensus, antenatal corticosteroids would be of benefit even if the rate of neonatal sepsis to double $5 \%$ to $10 \%$, if the index of intraventricular hemorrhage was decreased to $10 \%$, antenatal corticosteroids would remain beneficial although the rate of neonatal sepsis is increased by $50 \% .^{51}$

Coinciding with Gardner et al. ${ }^{51}$ believe that available evidence currently holds the recommendation from the Consensus on administering corticosteroids to patients with RPM between 24 and 34 weeks of gestational age. The potential benefits of a still small reduction in the incidence of intraventricular hemorrhage are superior to an unproven increase in the incidence of neonatal sepsis.

Therefore, acquire a huge importance the findings from the Consensus of 1994 in which significantly reduced incidences of syndrome of respiratory distress, necrotizing enterocolitis, intraventricular haemorrhage, perinatal mortality, etc, in association with antenatal administration of corticosteroids. The recommendations of the consensus are very important and we will mention them same as well as their conclusions:

\section{Recommendations}

A. Benefits of the administration of corticosteroids to fetuses at risk for preterm birth outweigh the potential risks of the medication.

B. All fetuses between 24 and 34 weeks of gestational age at risk of preterm birth should be considered candidates to receive antenatal corticosteroids.

C. The decision on the use of corticosteroids should not be modified by fetal sex or race or the availability of surfactant in UTI.

D. Patients with indication of tocolysis, have indication of corticoids.

E. The treatment consists of two doses of betamethasone (12mg) given via IM every 24 hours or dexamethasone $(6 \mathrm{mg})$ in four-dose via IM every 12 hours. The maximum benefit is obtained after 24hours after the start of the medication and its effect lasts appro- 
ximately for 7 days. Because a treatment less than 24 hours is also associated with significant reduction of neonatal mortality, respiratory distress, and intraventricular hemorrhage, corticosteroids should be equally given unless the birth is imminent.

In the case of RPM with gestational age less than 32 weeks and in the absence of amnionitis, it is recommended the use of steroids because of the high risk of intraventricular hemorrhage at this gestational age. In pregnancy with complications, where the birth before 34 weeks is probable, the use of corticosteroids is recommended unless there is evidence that steroids have an adverse effect on the mother or that the birth is imminent.

\section{Conclusion}

The treatment reduces the frequency of the respiratory distress syndrome, intraventricular hemorrhage and neonatal death, although to a lesser extent than with intact membranes. It is not clear if this treatment increases the possibility of maternal or neonatal infection.

However, the risk of death by prematurity is greater than the risk of infection. The dissemination of this information is of the utmost importance on the basis of the results presented and also to induce the change of attitude by professionals, since despite the fact that the benefits of steroids are well established, with exceptions their use even in critical situations does not exceed $40 \%$ of the cases in our midst.

An interesting study by Doyle et al ${ }^{57}$ showed that in the United Kingdom the use of corticoids spent $60 \%$ of the cases of potential use in 1991 to not less than $90 \%$ of use after 1994. In the discussion of this research the authors point out that the change of attitude was due largely to the dissemination of the benefits of the administration of corticosteroids by the Royal College of Obstetricians and Gynecologists.

However there are some features associated with the use of corticosteroids that should be present. Recently had been described considerable reductions in fetal breathing movements and fetal heart rate variability associated with the administration of betamethasone in the habitual doses to induce fetal lung maturation..$^{58-62}$ Using dexamethasone, a small increase in the variability of the FCF has been found except when there were associated with preeclampsia or a delay of growth. ${ }^{59}$

Mulder et al.$^{60}$ reported a randomized study to evaluate the effect on fetal functions of betamethasone and dexamethasone. A significant increase was found in the variability of long and short term after two doses of dexamethasone, regardless of associated pathology. There was also observed an increase in the variability associated with the administration of betamethasone, but in this case only lasted about 24hours, for then decrease during approximately for 48 hours. With respect to respiratory movements, these disappeared almost completely after the administration of betamethasone, while a reverse, a discreet increase was observed associated with the administration of dexamethasone.

These effects on fetal behavior seem to be mediated by a greater affinity of betamethasone by receptors in the central nervous system and also one plasma half-life greater than of the dexamethasone. Apparently not only side effects of betamethasone are more powerful but also its therapeutic effects. Both drugs have proven effective in reducing respiratory distress syndrome, but reductions in neonatal mortality only have been associated with betamethasone. ${ }^{61}$
It has been reported also that the babies of pregnant medicated with corticoids have arterial pressure values higher than controls and even in adult stages, would be more predisposed to develop alterations hypertensive. ${ }^{63}$ Although these effects occur, the benefits of treatment with corticoids of pregnancies between 24 and 34weeks of gestational age with threat of preterm delivery are obvious, reason that limits use even in situations is recommended as the case of a patient who enters in preterm labor. Since it has been shown that since the $4 \mathrm{hs}$ from administration the steroids begin to exert their effects, ${ }^{50}$ it is useful to delay the inevitable birth a few hours to result in action on the fetal lung of the corticosteroids.

An alternative, in which our group has been working, is in the administration intra amniotic of surfactant in cases of preterm labor that does not give time enough for the administration of corticosteroids. First of all, the experience was conducted in guinea pigs to determine if indeed the surfactant administered intra-amniotic reached the fetal lungs, this was duly substantiated (Figure 1). ${ }^{64}$

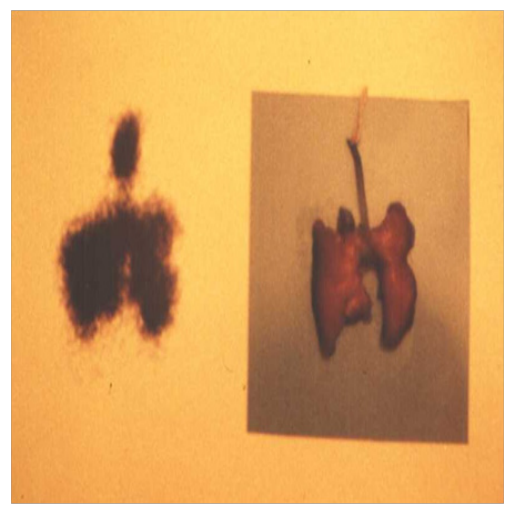

Figure I In this picture, we show the respiratory structures of the fetus (blue arrow) and the isotopic activity obtained in the gammagraphic evaluation (Green arrow). This last picture is equal so the anatomy of the fetal lungs meaning that the surfactanct with the Tc 99 reached the fetal lungs.

Then, the experience was made with preterm lambs at 120days of gestation (equivalent to 30 to 32 weeks of gestation in humans) observing the performance of ventilation of the lambs received surfactant before birth, was much better than those who received placebo. ${ }^{65}$ According to these studies, it is possible to manage surfactant in the amniotic fluid of patients with cervical dilatation enough preterm labor as to not allow time to implement the treatment with corticosteroids (Figure 2).

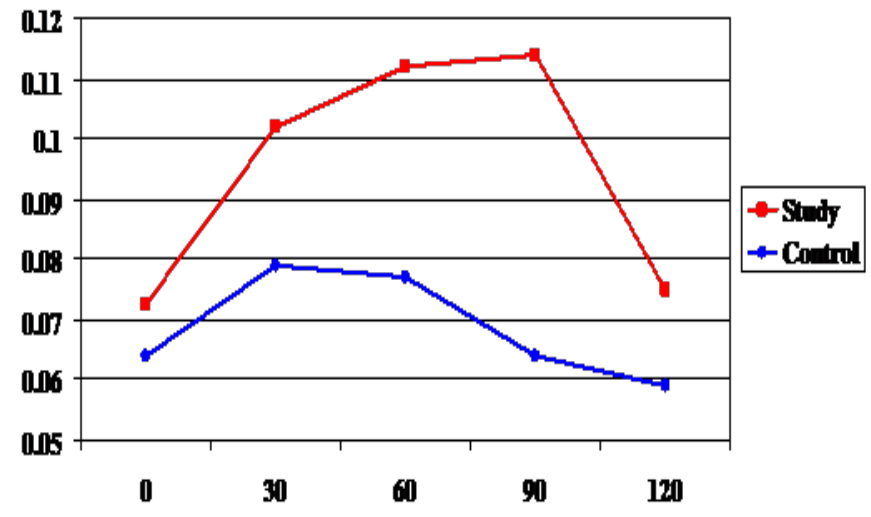

Figure 2 Antenatal Surfactant Administration Respiratory Outcome:ArterioAlveolar Ratio (y/y) Mean - SD. 


\section{Antibiotics}

In 1988, Amon et al. ${ }^{66}$ analyzed the usefulness of prophylactically administering ampicillin patients with pregnancy of preterm labor and associated RPM. At 72 hours of randomization risk of birth was 33\% in the group treated versus $50 \%$ in controls. The risk of birth a week later was $53 \%$ in the group treated versus $71 \%$ in controls. Conclude the authors the use of ampicillin can prolong gestation, reduce the incidence of neonatal infection and could reduce neonatal mortality.

In 1990 Johnston et al. ${ }^{67}$ conducted a prospective, double-blind study, on the administration of antibiotics in pregnancy preterm and RPM. The patients who received antibiotics (mezlocillin of load and ampicillin until birth) presented incidences of chorioamnionitis and endometritis lower than controls. The pathological studies of the placenta showed a lower incidence of histological amnionitis in the treated group, the latency period was greater in the treated group. The clinical suspicion of neonatal sepsis, respiratory distress syndrome, intraventricular hemorrhage, perinatal mortality and prolonged hospitalization were increased in the control group.

However, in 1992 Christmas \& cols ${ }^{68}$ reported a randomized study in a control group and other medicated with antibiotics (ampicillin, gentamicin, clindamycin, amoxicillin). The authors concluded that in the absence of evidence of specific maternal or fetal benefit with respect to broad-spectrum antibiotics, it seems inappropriate to use the recommendation.

Recently Egarter et $\mathrm{al}^{4}{ }^{4}$ performed a meta-analysis of seven randomized studies to estimate more precisely the effect of antibiotic prophylaxis on neonatal mortality, clinical sepsis in the newborn, necrotizing enterocolitis, intraventricular hemorrhage, and respiratory distress syndrome.

Among the 657 patients, antibiotic treatment significantly reduced the risk of neonatal sepsis and intraventricular hemorrhage. There was a significant effect on neonatal mortality, respiratory distress or necrotizing enterocolitis syndrome. The results of this meta-analysis show an improvement in neonatal morbidity in patients with prenatal preterm and RPM pregnancies treated with a different antibiotic regimens.

A variable of confusion in these assessments is to determine the role of an association of antibiotics with prenatal corticoids. For this purpose, Lovett \& $\operatorname{cols}^{69}$ evaluated a total of 112 patients medicated with antibiotics and corticoids. Prophylaxis with antibiotics and corticoids significantly decreased the total frequency of neonatal mortality, sepsis and respiratory distress syndrome, increasing significantly the birth weight compared with the group that only received corticoids.

So in summary, it seems to be properly documented that antibiotic treatment associated with corticosteroids is required for prevention of neonatal pathologies clearly specified. The issue to be resolved is which is the best antibiotic scheme to introduce.

\section{Group B streptococcus}

Streptococcus Group B (EB) is a pathogen in pregnant women and infants. They are present in $15 \%$ to $40 \%$ of pregnant women and are one of the main causes of neonatal sepsis and a significant cause of chorioamnionitis and endometritis after delivery. The incidence of neonatal sepsis by EB is close to approximately 2 to 4 newborns per 1,000 live births. In association with sepsis by EB high-risk conditions include maternal colonization, preterm birth, prolonged rupture of the membranes and intrapartum maternal fever. The American Academy of Pediatrics and the American College of Obstetricians and Gynecologists ${ }^{70}$ made recommendations for prevention of neonatal sepsis by EB suggesting antibiotics in patients with EB and preterm delivery, intrapartum maternal fever and more than 18 hours RPM.

Regan et $\mathrm{al} . .^{70}$ conducted a prospective study to evaluate the influence of maternal colonization by EB and perinatal outcome. EB colonization was associated with an increased risk of preterm birth and low weight at birth. However the colonization with EB of vagina and cervix was not a reliable element to predict neonatal sepsis. The colonization at the time of birth was associated with neonatal sepsis.

Grable et al. ${ }^{71}$ conducted a randomized study to determine if ampicillin prolongs the period of latency after RPM in patients colonized by EB. The use of ampicillin increased the percentage of positive culture for EB patients who remained pregnant after 48 hours of produced the RPM. Ampicillin can provide an opportunity to implement the corticoid treatment to allow the prolongation of pregnancy for at least 48 hours.

Even a recent communication by Bloom et al. ${ }^{72}$ shows that bactericidal levels of ampicillin may be obtained quickly in fetal blood and amniotic fluid after an intravenous dose of 2 grams administered to the mother for prophylaxis of neonatal pathology.

As we will see later, in our service is performed routine cultivation of material obtained from vagina, cervix and rectum if RPM preterm for the detection of different germs, including the EB.

\section{Macrolides-azithromycin}

It has been mentioned in previous paragraphs the possibility of a role in the ovular infection germs as Mycoplasma hominis, Ureaplasma urealiticum and Chlamydia trachomatis. In the author's experience, on the basis of data not published and not controlled, the use of this antibiotic in patients with a history of preterm birth and/ or substantial cervical modifications in the current pregnancy, it has resulted in prolongation of pregnancy up to gestational ages where neonatal evolution is presented as more benign.

Even in cases where the pathology of perinatal loss earlier reported chorioamnionitis, the empirical use of macrolides from the 12 weeks of gestation until 34 weeks, appear with a favorable perinatal outcome in all cases.

While the quality of this information is not the best for being personal observation not controlled, the treatment success is striking and the potential importance of macrolides is demonstrated in the concretion of the ORACLE project. Several works have been reported with the use of macrolides to evaluate their potential role in the treatment of the threat of preterm delivery and the RPM. One of the problems is the doses used, since most of the studies used small doses compared with the commonly used to treat other cases with these antibiotics. It has no sense to evaluate the impact of erythromycin using doses lower than two grams day. For example, the dose selected of erythromycin is ridiculous in the ORACLE study, as much as I am not going to analyze it. Just I am going to say that is weird to expect an effective therapeutic effect in adults implementing an erythromycin dose that nobody would use to treat a flu in a child.

To evaluate the effectiveness of oral erythromycin in the extension of the period of latency and reduction of infectious morbidity after 
RPM, Mercer et al. ${ }^{73}$ did a randomized study to receive erythromycin $333 \mathrm{mg}$ every 8 hours or placebo. He had observed that oral erythromycin in these doses postposed but did not prevent the start of clinical infection and there was no decrease in neonatal morbidity and mortality. Also McGregor et al. ${ }^{74}$ had evaluated in a prospective and randomized study to receive $333 \mathrm{mg} / 8$ hs or placebo.

In this study treatment with erythromycin was well tolerated and associated with prolongation of pregnancy and reduction in neonatal intensive care requirements. In the literature there is no found controlled studies with adequate doses of antibiotics designed to assess the impact of the use of macrolides in suspicion of ovular infection with intact membranes or not. In addition, it should be noted what would happen if the antibiotic employed is a macrolide of latest generation such as azithromycin, clarithromycin, etc.

There seems to be agreement on the importance of ovular infection as etiologic agent associated with preterm birth (with membranes intact or broken). But we must not forget that more or less evenly, studies found an incidence of histological chorioamnionitis between 40 and $60 \%$ of the analyzed cases. This fact would make it possible to infer that approximately two thirds of prematurity could be associated to ovular infection, especially in cases of prematurity before 30 weeks of gestational age.

An example of the need to treat the infections caused by these germs (Chlamydia-Mycoplasma-Ureaplasma) during pregnancy is a recent study about the use of doxycycline during pregnancy. Czeizel $\&$ Rockenbauer ${ }^{75}$ analyzed the doxycycline teratogenic risk during pregnancy. The results show that there were very few risks to the fetus, so if this antibiotic treatment is deemed necessary it seems there would have no contraindication for use.

\section{Treatment}

To the approach of treatment and follow-up of cases of RPM, we will expose to follow-up standard for the existing RPM at different gestational ages in the service of obstetrics at the Hospital Alemán of Buenos Aires:

\section{Less than 20weeks pregnant}

Extramembranous pregnancy. The possibilities for the fetus are minimal. Maternal antibiotic coverage with ampicillin sulbactam $750 \mathrm{mg}$ every 12 hours for 10 days and azithromycin $500 \mathrm{mg}$ per day for 72 hours is indicated. These courses of antibiotics may reiterate if deemed necessary.

If the patient spontaneously start the expulsion, tocolysis will not be administered.

\section{Pregnancy do 20 to 26 weeks}

The bigger the gestational age within this segment, greater is the probability of fetal survival.

Proceed to cultivate material endocervical, vaginal and anal oriented to detection of aerobic, anaerobic and particularly Mycoplasma, Chlamydia and Ureaplasma, EB. Then starts the administration of amoxicillin $750 \mathrm{mg}$ every 12 hours for 10days and azithromycin 500mg per day for 72 hours according to the guidelines of the CDC.

Once this treatment is finished and if the first crop turned out positive for $\mathrm{EB}$, it is cultivated again to assess the situation. If the first crop or the latter is negative, not reiterates the course of amoxicillin.
The series of azithromycin, are repeated every 7 days 72 hours. From 24 weeks gestational age, begins the induction of fetal lung maturation with betamethasone, 24 in 48hours in two applications of $12 \mathrm{mg}$ each $\mathrm{mg}$ (acetate $6 \mathrm{mg}$ and $6 \mathrm{mg}$ phosphate). Regardless of the time of the dose of attack, if the patient starts labor before the inducccion in less than 34 weeks pregnancy or childbirth, indicated a new dose of betamethasone.

If start spontaneous dynamics before 24 weeks, is left to evolve. After 24weeks, will attempt to tocolysis with intravenous betamimetics for 12 hours and indomethacin (oral every 12 hours or via anal every 24hours) for 72 hours.

\section{7-34weeks pregnant}

Follow the same antibiotic scheme and the same procedure according to crops than in the previous case.

Similar schemes will also be used for the induction of fetal lung maturation.

If spontaneous uterine dynamics is installed, is implemented as systematic tocolytic.

\section{More than 34weeks pregnant}

If the case comes with RPM's lower gestational ages, tocolytic agents, corticosteroids, are suspended at 34 weeks and remains the antibiotic scheme adopted. After 72 hours of met 34 weeks, indicated the induction of parturition.

If the patient enters with RPM and a gestational age between 34 and 36weeks, crops are made, antibiotic therapy is started and within 48hours of the RPM is indicated the induction of parturition.

If the patient enter with RPM between 36 and 37.6weeks gestational age, antibiotic therapy is initiated and indicated the induction of parturition within $24 \mathrm{hs}$ from RPM.

If the patient enter with RPM and an equal or greater than 38 weeks gestational age, induction is indicated between 12 and 24 hours from RPM.

\section{General following}

i. Will be checked on a daily basis (3times a day) the maternal axillary temperature.

ii. Apply for white blood cell count every 48 to 72 hours. The increase in the count that occur after the administration of corticosteroids should be taken into account

iii. If this is the case and the patient is afebrile, in the absence of uterine dynamics occurs a curve of evolution of WBCs in daily frequency. In the absence of ovular infection, you will notice a decrease in count in 48 to 72 hours. If this decrease does not occur, shall be considered as probable ovular infection and will proceed to discuss within the scope of the perinatal team decision to adopt.

iv. Prohibition of vaginal exams.

v. Patient at absolute rest.

vi. Weekly evaluation of amniotic fluid volume and from 26week's simultaneously weekly ultrasound for assessing fetal breathing movements with technique of Manning (at least a series of uninterrupted movements of 30-second in a span of 30minutes). 
vii. Starting from week 30 , adds the fetal monitoring weekly and every 72 hours after the 32 nd week. In all cases of monitoring doubtful, the presence of fetal breathing movements should be deemed sufficient reinsurance of fetal health. In the case of no reactivity, it should be suspected possible ovular infection and be increased prevention in this connection.

viii. If the patient has culture-positive for EB, previous newborn inpatient in NICU for sepsis to EB or pregnant woman presents intrapartum hyperthermia, intravenous ampicillin will be started at the beginning of labour in initial dose of $2 \mathrm{~g}$ and then 1 to $2 \mathrm{~g}$ every 4 to 6hours until the birth.

\section{Amnioinfusion}

In 1989 Imanaka et al. ${ }^{63}$ reported continuous infusion by catheter in patients with RPM before term. They infused saline solution continuously at a rate of $10-20 \mathrm{ml}$ per hour. The lakes of amniotic fluid measured before the procedure $2.7 \mathrm{~cm}$ and after the same more than $5 \mathrm{~cm}$

At the Hospital Alemán Obstetrics Service, we use this resource in patients with pregnancies of less than 34weeks and severe oligohydramnios with or without RPM. The goal is avoid or mitigate primarily pulmonary hypoplasia resulting from not having liquid to swallow, given that this seems to be one of the mechanisms involved in the development of fetal lungs. ${ }^{76}$ Also avoids postural deformations by fetal compression, the funiculars deceleration also by compression and sufficient contrast is generated to correctly evaluate both the health and fetal anatomy by ultrasound.

We do not perform continuous but repeated infusions, a 200 cc of saline solution at $37^{\circ} \mathrm{C}$ of temperature for time, one infusion every 3 to 5 days according to the need, evaluating the need for depending on the volume of amniotic fluid lakes. It is the procedure with or without RPM when the lakes are smaller than $2 \mathrm{~cm}$ in each quadrant. After the procedure is seen an immediate increase in the volume of these lakes (increase 4 to $6 \mathrm{~cm}$ ), begins a general major fetal activity and continuous fetal breathing movements. To avoid uterine contractility, to perform the procedure under tocolysis with betamimetic therapy and is performed with azithromycin antibiotic coverage. We evaluate the situation with a daily ultrasound monitoring. Usually 24-48hours of infusion, the lakes have decreased is size, but there was increasing the gastric chamber and the fetal bladder. Return the size of the lakes of amniotic fluid to the initial volume, indicates the need to repeat the procedure and repeat the same sequence of events.

We have performed in our service and in the private practice several procedures of amnioinfusion with good results. Neonatal evolution has always been very good, with substantial decreases the need for ventilatory support despite extreme prematurity of some of the cases assisted. All cases received also induction of fetal lung maturation with corticoids according to usual doses with betamethasone.

In cases of extreme oligohydramnios, amnioinfusion is a therapeutic resource important to benefit fetal lung development that should be present because their few complications and the apparent benefits. There have been no controlled studies on this method, so it is recommended prudence in the choice of the case and its execution, and eventually, to keep it within the scope of the investigation.

\section{Complications}

While the main complication associated with RPM is perinatal infection, there are other important also complications that we will analyze.

Prematurity: There is a strong association between RPM and preterm birth. According to the study by Verber et al. ${ }^{77}$ RPM incidence was $3 \%$ after 32 weeks, $28 \%$ between 28 and 31 weeks and $31 \%$ before 28 weeks of gestational age. Other authors have found similar associations. $^{78}$

The degree of prematurity of the newborn alone is not associated with increased neonatal mortality to lower gestational age, they should be deemed the morbidity associated with extreme prematurity. According to Hack \& $\operatorname{cols}^{79}$ was considered serious neonatal morbidity cases with at least one of the following factors: chronic lung disease, necrotizing enterocolitis, or a grade III or IV intraventricular hemorrhage. The incidence of morbidity in survivors according to the weight at birth was $55 \%$ in newborns of less than $751 \mathrm{~g}, 39 \%$ in 751 $1.000 \mathrm{~g}, 26 \% 1,001$ to $1,250 \mathrm{~g}$ and $14 \%$ between 1251 and $1,500 \mathrm{~g}$.

Conversely, disease-free survival has been communicated by Lefebvre et al..$^{80}$ in figures of $44 \%$ for born at 24 weeks, $71 \%$ at 25 weeks, $57 \%$ at $26,76 \%$ at 27 and $72 \%$ at 28 weeks. Kilpatrick et al. ${ }^{81}$ recently reported that survival at 24 weeks was only $43 \%$ despite treatment with corticoids and surfactant. The cost for a survivor born at 24 weeks was greater than the cost of those born after one week in utero. Results improved markedly between 24 and 26weeks and small differences in gestational age were reflected in economical differences. Concluded the authors that all efforts should be done on the prolongation of pregnancy.

The same group of researchers, Piecuch $\& \operatorname{cols}^{82}$ analyzed the neurological development of the infants between 24 and 26weeks. The number of neurologically normal children from birth to 26weeks (90\%) was similar to those born between the 27 and 32weeks. Although children born at 24 and 25 weeks did not show an incidence significantly greater than cerebral palsy, the number of children suspected of suffering some kind of injury to the year of life is important among those born at 24 weeks.

The number of babies born at 24 and 25 weeks who presented severe developmental disorders was important. These data suggest that a mother in labor for at 24 weeks, the chance of having a child with normal intellect or borderline to survive is $26 \%$, if gestational age is 25 weeks this chance rises to $52 \%$ and 26 weeks the likely increase is $74 \%$.

\section{Pulmonary hypoplasia and fetal deformities}

These are complications associated with the prolonged oligohydramnios. According to the study by Thibeault et al. ${ }^{83}$ in patients with severe oligohydramnios and positional deformities, 20 of 21 cases were pulmonary hypoplasia with a frequency of perinatal mortality of $86 \%$. In total the perinatal mortality in the group without deformities was much smaller $(24 \%)$. The duration of the severe oligohydramnios and gestational age at the RPM are independent predictors of perinatal risks.

A severe oligohydramnios of more than 14days of evolution and the RPM before 25 weeks are associated with neonatal mortality of more than $90 \%{ }^{84,85}$ Apparently the implementation of the lung area and the relationship of the thoracic circumference on the abdominal obtained through ultrasound would be useful elements to assess pulmonary hypoplasia. ${ }^{86}$

These patients are also at risk of accidentally cord prolapse, 
increased risk of placental abruption (especially cases of RPM with bloody fluid loss) and severe alterations of fetal growth. ${ }^{87,88}$

\section{Prevention}

Measures to decrease the incidence of RPM before the term are directly related to those aimed to reduce prematurity associated with infectious factors. In general, treatment of bacterial vaginosis in the gynecologic patient who can become pregnant is of most importance.

This attitude involves the appropriate use of antibiotics such as doxycycline and macrolides (azithromycin, clarithromycin, Roxithromycin, etc) and shoul be taken in account the imidazolic ones. The same approach should apply for the appropriate treatment of pelvic inflammatory disease where these antibiotics should be part of the first therapeutic line.

Bacterial vaginosis can be treated during gestation with vaginal imidazolic and systemic macrolides. Do not take this conduct, means to let the patient at risk of acquiring any of the complications treated in this chapter.

At the present time of Obstetrics and Perinatology, much of the complications associated with prematurity, membranes intact or broken, are associated with a particular group of germs and infectious factors. There is no study that had documented a clear deleterious effect generated by antibiotic treatment with macrolides during pregnancy so far. In the author's opinion, there is no data that would justify depriving of the potential beneficial effects of this treatment patients pregnant at high risk of infection by Mycoplasma, Ureaplasma and Chlamydia. For the specific case of EB, cultivation and treatment guidelines laid down in the rules of monitoring intended to prevent transmission of this infection should be followed.

\section{Acknowledgments}

None.

\section{Conflict of interest}

Author declares that there is no conflict of interest.

\section{References}

1. Iams J. Premature birth. In: Iams J, Zuspan F, editors. Manual of obstetrics and gynecology. USA: Mosby Year Book; 1990. 125-136p.

2. Illia R. Standard for the assistance of the RPM. Obstetricia.1.996 service. 1995 .

3. Guinn DA, Goldenberg RL, Hauth JC, et al. Risk factors for the development of preterm premature rupture of the membranes after arrest of preterm labor. Am j Obstet Gynecol. 1995;173(4):1310-1315.

4. Egarter $\mathrm{C}$, Leitich $\mathrm{H}$, Karas H. Antibiotic treatment in preterm premature rupture of membranes and neonatal morbidity. Goal analysis. $A m j$ Obstet Gynecol. 1996;174:589-97.

5. Fortunato SJ, Menon R, Lombardi SJ. Collagenolytic enzymes (gelatinases) and their inhibitors in human amniochorionic membrane. $A m$ Obstet Gynecol. 1997;177(4):731-741.

6. Hampson V, Liu D, Billett E, et al. Amniotic membrane collagen content and distribution in women with preterm premature rupture of the type membranes in pregnancy. BR $j$ Obstet Gynaecol. 1997;104(9):1087-1091.

7. Romero R, Quintero R, Oyarzun E, et al. Intraamniotic infection and the onset of labor in preterm premature rupture of membranes. Am j Obstet Gynecol. 1988;159(3):661-666.
8. Romero R, Mazor M. Infection and preterm labor. Clin Obstet Gynecol. $1988 ; 31: 553-584$

9. Grandi C, Fuksman R, Garcia H. Clinicopathological relationship between ovulate infection and preterm birth. Journal of Hospital M.I.R.Sarda. 1993;11:13-16.

10. Illia R, Valenti E. Diagnosis of Perinatal situation. Journal of the M.i.r.sarda Hospital. 1988;1:14-24.

11. Arias F, Victoria A, Cho K, et al. Placental histology and clinical characteristics of patients with preterm premature rupture of membranes. Obstet Gynecol. 1997;89(2):265-271.

12. Murtha AP, Greig PC, Jimmerson CE, et al. Maternal serum interleukin 6 concentrations in patients with preterm premature rupture of membranes and evidence of infection. Am j Obstet Gynecol. 1996;175(4 Pt 1):966-969.

13. Lavery J, Miller C. Deformation and creep in the human chorioamniotic sac. Am j Obstet Gynecol. 1979;134(4):366.

14. Toppozada MK, Sallam NA, Gaafar AA, et al. Role of repeated stretching in the mecanism of timely rupture of the membranes. Am j Obstet Gynecol. 1970;108(2):243.

15. Skinner S, Higgins G. Collagen content on human amniotic membranes: Effect of gestational length and premature rupture. Obstet Gynecol. 1981;57:487.

16. Hills B, Cottno D. Premature rupture of membranes and surface emergency: Possible role of surfactant. Am j Obstet Gynecol. 1984;149:896.

17. Schoonmaker JN, Lawellin DW, Lunt B, et al. Bacteria and inflammatory cells reduces chorioamniotic membrane integrity and tensile strengh. Obstet Gynecol. 1989;74(4):590-596.

18. Mc Gregor J, Scoonmaker J, Hunt D. Antibiotic inhibition of bacterially induced fetal membrane weakening. Obstet Gynecol. 1990;76:124.

19. Gauthier DW, Meyer WJ. Comparison of Gram stain, leukocyte estearase activity and amniotic fluid glucose concentration in predicting amniotic fluid culture results in preterm premature rupture of membranes. Am j Obstet Gynecol. 1992;167(4 Pt 1):1092-1095.

20. Romero R, Yoon B, Mazor M, et al. A comparative study of the diag nostic performance of amniotic fluid glucose, white blood cell count, Interleukin 6 and Gram stain in the detection on microbial invasion in patients with premature rupture of membranes. Am j Obstet Gynecol. 1993;169(4):839-851.

21. Sweet R, Launders D, Walder C, et al. Chlamydia Trachomatis infection and pregnancy outcome. Am j Obstet Gynecol. 1987;156:824-833.

22. Vile Y, Carroll SG, Watts P, et al. Chlamydia Trachomatis infection in prelabour amniorrhexis. BRj Obstet Gynaecol. 1997;104(9):1091-1093.

23. Mc Gregor J, French J. Chlamydia Trachomatis infection during pregnancy. Am J Obstet Gynecol. 1998;164(Suppl 1):6.

24. Gravett MG, Haluska GJ, Cook MJ, et al. Fetal and maternal endocrine responses to experimental intrauterine infection in rhesus monkeys. $\mathrm{Am}$ j Obstet Gynecol. 1996;174(6):1725-1731.

25. Buschamann A, Bisson S. Interleukin 8 in urine: a new diagnostic parameter for intra-amniotic infection after premature rupture of the membranes. BR j Obatet Gynaecol. 1997;104(4):499-502.

26. Elliot B, Brumham R, Laga M, et al. Maternal gonococcal infection as a preventable risk factor for low birth weight. $J$ Infect Dis. 1990;161(3):531-536.

27. Minkoff H, Grunebaum A, Schwarz R, et al. Risk factors for prematurity and premature rupture of the membranes. A prospective study of the vaginal flora in pregnancy. Am j Obstet Gynecol.1984;150:965-972. 
28. Gravett MG, Nelson HP, DeRouen T, et al. Independent associations of bacterial vaginosis and Chlamydia Trachomatis infection with adverse pregnancy outcome. JAMA. 1986;256(14):1899-1903.

29. Newton E, Piper J, Peairs W. Bacterial vaginosis and intraamniotic infection. Am j Obstet Gynecol. 1997;176:672-677.

30. Fargason C, Peralta M, Rouse D, et al. The pediatric costs of strategies for minimizing the risk of early-onset group B Streptococcal disease. Obstet Gynecol. 1997;90(3):347-352.

31. Regan J, Klebanoff M, Nugent R, et al. Colonization with group B streptococci in pregnancy and adverse outcome. Am j Obstet Gynecol. 1996;174(4):1354-1360.

32. Ernst J, Meis P, Moore M, et al. Vaginal PH: marker of preterm premature rupture of the membranes. Obstet Gynecol. 1989;74(5):734-738.

33. Riedewald S, Krentzman, Heinde T, et al. Vaginal and cervical $\mathrm{Ph}$ in normal pregnancy and pregnancy complicated work. J Perinat Med. 1990;18(3):181-186.

34. Sikorski R, Juskiekowicz T, Paszkowski T. Zinc status in women with premature rupture of membranes at term. Obstet Gynecol. 1990;76(4):675-677.

35. Haaley C, Main D, Gabb S. Risk factors for preterm rupture of the fetal membranes. Am j Perinat. 1990;7(4):374-379.

36. Harger J, Hsing A, Tunola RE, et al. Risk factors for preterm premature rupture of fetal membranes. A multicenter case control study. Am j Obstet Gynecol. 1990;163(1 Pt 1):130-137.

37. Illia R, Valenti E. Perinatal diagnosis of preterm delivery. Journal of Hospital Sarda. 1988;1:14-24.

38. Illia $\mathrm{R}$, Leveque $\mathrm{R}$, Mayer $\mathrm{H}$, et al. Role of cervical cerclage and prolonged antibiotic therapy with azithromycinin in patients with previous perinatal loss by amnionitis. J Matern Fetal Neonatal. 2017;30(3):309-312.

39. Romero R, Mazor M. Infection and preterm labor. Clin Obstet Gynecol. 1988;31(3):553-584.

40. Mc Gregor J, French J, Richter R, et al. Cervico-vaginal microflora and pregnancy outcome: results of a double-blind placebo-controlled trial of erythromicin treatment. Am j Obstet Gynecol. 1990;163(1):580-1591.

41. Ryan G, Abdella T, McNeeley SG, et al. Chlamydia trachomatis infection in pregnancy and effect of treatment on outcome. Am j Obstet Gynecol. 1990;162(1):34-39.

42. Hsu C, Aversa K, Meaddough E, et al. Elevated amniotic fluid nitric oxide metabolites and cyclic guanosine monophosphate 3-5 in pregnant women with intraamniotic infection. Am j Obstet Gynecol. 1997;177(4):793-796.

43. Stiemer B, Buschmann A, Bisson, et al. Interleukin 8 in urine: a new diagnostic parameter for intra-amniotic infection after premature rupture of the membranes. BR j Obstet Gynaecol. 1997;104(4):499-502.

44. Vintzileos A, Campbell W, Nochimson D, et al. Fetal biophysical profile versus amniocentesis in predicting infection in preterm premature rupture of the membranes. Obstet Gynecol. 1986;68(4):488-494.

45. Vintzileos A, Campbell W, Nochimson DJ et al. The fetal biophysical profile in patients with premature rupture of the membranes. An early predictor of fetal infection. Am j Obstet Gynecol. 1985;152(5):510-516.

46. Vintzileos AM, Bors-Koefoed R, Pelegano J, et al. The use of fetal biophysical profile improves pregnancy outcome in premature rupture of the membranes. Am j Obstet Gynecol. 1987;157(2):236-240.

47. Gilstrap L, Levenno K, Cox S, et al. Intrapartum treatment of acute chorioamnionitis: impact on neonatal sepsis. Am $j$ Obstet Gynecol. 1988;159(3):579-583.
48. Spelling RS, Ramamurthy RS, Gibbs RS. A comparison of intrapartum versus immediate postpartum treatment of intraamniotic infection. Obstet Gynecol. 1987;70(6):861-865.

49. Quinn P, Butany j, Taylor J, et al. Chorioamnionitis: Its association with pregnancy outcome and microbial infection. Am j Obstet Gynecol. 1987;156(2):379-387.

50. Crowley P. Antenatal corticosteroid therapy: a meta-analysis of the randomized trials, 1972 to 1994. Am $j$ Obstet Gynecol. 1995;173(1):322-335.

51. Gardner M, Papile L, Wright L. Antrenatal corticosteroids in pregnancies complicated by preterm premature rupture of membranes. Obstet Gynecol. 1997;90(5):851-853.

52. Wright L, Horbar J, Gunkel H, et al. Evidence from multicenter networks on the current use and effectiveness of antenatal corticosteroids in low birth weight infants. Am j Obstet Gynecol. 1995;173(1):263-269.

53. Gunkel J, Mitchell B. Observational evidence for the efficacy of antenatal steroids from randomized studies of surfactant replacement. $A m j$ Obstet Gynecol. 1995;173(1):281-285.

54. Wright L, Verter J, Younes N, et al. Antenatal corticosteroid administration and neonatal outcome in very low birth weight infants: The NICHD Neonatal Research Network. Am j Obstet Gynecol. 1995;173(1):269-274.

55. Morales WJ, Diebel ND, Lazar AJ, et al. The effect of antenatal dexamethasone administration on the prevention of respiratory distress syndrome in preterm gestations with premature rupture of the membranes. Am j Obstet Gynecol. 1986;154(3):591-595.

56. Chapman P. Lack of apparent benefit in corticosteroid $<1,000 \mathrm{~g}$ infants born after preterm amnion rupture. Am j Obstet Gynecol. 1996;174:316.

57. Doyle M, Hamilton R, Johanson R, et al. The use of corticosteroids for maduration of fetal lungs in patients with preterm prelabour rupture of the membranes. BR j Obstet Gynaecol. 1994;101(12):1078-1079.

58. Derks J, Mulder E, G Visser. The effects of maternal bethametasone administration on the fetus. BR j Obstet Gynaecol. 1995;102(1):40-46.

59. Dawes G, Serra-Serra V, Moulden M, et al. Dexamethasone and fetal heart rate variation. BR j Obstet Gynaecol. 1994;101(8):675-79.

60. Mulder E, Derks J, Visser G. Antenatal corticosteroid therapy and fetal behavior: a randomized study of the effects of bethametasone and dexamethasone. BR j Obstet Gynaecol. 1997;104(1):239-247.

61. Ballard P, Ballard R. Scientific basis and therapeutic regimes for use of antenatal glucocorticoids. Am j Obstet Gynecol. 1995;173(1):254-262.

62. Wallace EM, Chapman J, Stenson B, et al. Antenatal corticosteroid prescribing: setting standards of care. BR $j$ Obstet Gynaecol. 1997;104(11):1262-1266.

63. Imanaka M, Ogita S, Sugana T. Saline solution amnioinfusion for oligohydramnios after premature rupture of the membranes. A preliminary report. Am j Obstet Gynecol. 1989;161(1):102-106.

64. Illia R, Solana C, Oliveri P, et al. Evidence of fetal pulmonary aspiration of intra-amniotic surfactant in animal administered Experiment. Journal of Perinatal Medicine. 2004;32(4):354-358.

65. Ricardo Illia CS, William L, Matias UI, et al. Prenatal Intraamniotic Surfactant Administration Improves Lung Function At Birth And The Effects Of Conventional Postdelivery Treatment In Animal Model. Journal of Gynecology and Neonatal Biology. 2016;2:1-4

66. Amon E, Lewis S, Sibai B, et al. Ampicillin prophylaxis in preterm premature rupture of the membranes: A prospective randomized study. Am j Obstet Gynecol. 1988;159:539-543. 
67. Johnston M, Sánchez Ramos L, Vaughn AJ, et al. Antibiotic therapy in preterm premature rupture of membranes: A randomized, prospective, double blind trial. Am j Obstet Gynecol. 1990;163(3):743-747.

68. Christmas J Cox, Andrews W. Expectant management of preterm ruptured membranes: Effects of antimicrobial therapy. Obstet Gynecol. 1992;80:759-762.

69. Lovett S, Weiss J, Diogo M, et al. A prospective, double blind, randomized, controlled clinical trial of ampicillin-sulbactam for preterm rupture of membranes in women receiving antenatal corticosteroid therapy. Am j Obstet Gynecol. 1997;176(5):1030-1038.

70. Regan JA, Klebanoff MA, Nugent RP, et al. Colonization with group B streptococci in pregnancy and adverse outcome. Am j Obstet Gynecol. 1996;174(4):1354-1360

71. Grable I, Garcia P, Perry D. Group B Streptococcus and preterm premature rupture of membranes: A randomized, double blind clinical tria of antepartum ampicillin. Am j Obstet Gynecol. 1996;175:1036-1042.

72. Bloom SL, Cox SM, Bawdon RE, et al. Ampicillin for neonatal group B streptococcal prophylaxis: How rapidly can bactericidal concentrations be achieved?. Am j Obstet Gynecol. 1996;175(4 Pt 1):974-976.

73. Mercer B, Moretti M, R Prevost, et al. Erithromycin therapy in preterm rupture of the membranes: A prospective, randomized trial of 220 patients. Am j Obstet Gynecol. 1992;166(3):794-802.

74. McGregor J, French J, Seo K. Antimicrobial therapy in preterm premature rupture of membranes: results of a prospective, double blind, placebo controlled trial of erithromycin. Am j Obstet Gynecol. 1991;165:632-640

75. Czeizel AM, Rockenbauer M. Teratogenic study of doxicicline. Obstet Gynecol. 1997;89(4):524-528.

76. Illia R, C Solana, Oliveri P, et al. Fetal uptake of surfactant marked with Tc 99 injected by amniocentesis. Glorieux, Unpublished data; 1990.

77. Verber IG, Pearce JM, New LC, et al. Prolongued rupture of the fetal membranes and neonatal outcome. J Perinat Med. 1989;17(6):469.
78. Hoekstra R, Jackson J, Myers H. Improved neonatal survival following multiple doses of bovine surfactant in very premature neonates at risk for respiratory distress syndrome. Pediatrics. 1991;88:10.

79. Hack M, Horbar J, Mallor M, et al. Very low birth weight outcomes of the National Institute of Children Health and Human Development Neonatal Network. Pediatrics. 1991;87:587-597.

80. Lefebvre F, Gagnon T. Neonatal survival and disability rate at age 18 months for infants born between 23 and 28 weeks of gestation. Am j Obstet Gynecol. 1996;174(3):833-838.

81. Kilpatrick SJ, Schlueter MA, Piecuch R, et al. Outcome of infants born at 24-26 weeks gestation: I. Survival and cost. Obstet Gynecol. 1997;90:803-808.

82. Piecuch RE, Leonard CH, Cooper BA, et al. Outcome of infants born at 24-26 weeks gestation. II. Neurodovolopmental outcome. Obstet Gynecol. 1997;90(5):809-814.

83. Bealthy E, Hall R, Thibeault. Neonatal pulmonary hypoplasia with premature rupture of the fetal membranes and oligohydramnios. J Pediatr. 1985;107:273.

84. Kilbride H, Yeast j, Thibeault DW. Defining limits of survival: Lethal pulmonary hypoplasia after midtrimester premature rupture of membranes. Am j Obstet Gynecol. 1996;175(3 Pt 1):675-681.

85. Vergani P, Ghidini A, Locatelli A, et al. Risk factors for pulmonary hypoplasia in second-trimester premature rupture of membranes. $A m j$ Obstet Gynecol. 1994;170(5 pt 1):1359-1364.

86. Yoshimura S, H Masuzaki, Gotoh $\mathrm{H}$, et al. Ultrasonographic prediction of lethal pulmonary hypoplasia: Comparison of eight different ultrasonographic parameters. Am j Obstet Gynecol. 1996;175(2):477-483.

87. Darby M, Caritis S, Schwartz S. Placental abruption in the preterm gestation. An association with chorioamnionitis. Obstet Gynecol. 1989;74(1):88-92.

88. Illia R, De Diego, Dinerstein A. Analysis of biochemical and clinical parameters for the diagnosis of infection ovulate in prolonged rupture of membranes products of conception. Toko Gin Pract. 1994;53:98-101. 